\title{
The Human Brain Revisited: Opportunities and Challenges in Postmortem Studies of Psychiatric Disorders
}

\author{
David A. Lewis, M.D.
}

Studies of the postmortem human brain have become an increasingly essential element of the effort to understand the neurobiology of psychiatric disorders, especially in light of advances in our knowledge of functional brain circuitry and the new opportunities to apply the approaches of genomics and proteomics. This Perspective reviews some of the opportunities afforded by investigations of the postmortem human brain, and offers suggestions for improving the quality of future studies through the use of wellcharacterized brain specimens, well-constructed experimental designs and well-controlled confounds. [Neuropsychopharmacology 26:143-154, 2002] (C) 2002 American College of Neuropsychopharmacology. Published by Elsevier Science Inc.
KEY WORDS: Brain banks; Clinical diagnosis; Postmortem interval; Schizophrenia

Research into the pathophysiological mechanisms operative in a number of psychiatric disorders, and the insights that those findings provide regarding pathogenetic factors and treatment approaches, have been greatly accelerated in recent years through the increasing temporal, spatial and neurochemical resolution of in vivo neuroimaging techniques. In addition, genetic and behavioral animal models have provided tractable systems for investigating the details of plausible pathophysiological mechanisms. However, neither in vivo neuroimaging nor animal models permit the direct investigation of the diseased brain tissue.

Although disparaged in the past due to the limitations imposed by confounding variables and the lack of

From the Departments of Psychiatry and Neuroscience, University of Pittsburgh, Pittsburgh, PA

Address correspondence to: David A. Lewis, M.D., Department of Psychiatry, University of Pittsburgh, 3811 O'Hara Street, W1651 BST Pittsburgh, PA 15213, Tel.: (412) 624-3934, Fax: (412) 624-9910, E-mail: lewisda@msx.upmc.edu

Received August 27, 2001; revised October 12, 2001; accepted October 17, 2001. reproducible findings (Plum 1972), the use of postmortem human brain specimens in the study of psychiatric disorders has experienced new life in the past 15 years. The current interest in such studies may be traced to the confluence of several streams of thought. The first, and perhaps most critical, influence was the growing recognition in the 1960s and 1970s that major psychiatric disorders are diseases of the brain, and that at least some disorders, such as schizophrenia, are associated with altered brain anatomy (Stevens 1973; Johnstone et al. 1976). Second, the pioneering investigations, principally of schizophrenia, initiated in the 1980s by Francine Benes, Joel Kleinman, Arnold Scheibel and others, began to demonstrate that postmortem studies could incorporate the types of experimental designs and controls for potential confounds that characterized other areas of empirical study. Finally, the broad advances in our fund of knowledge in neuroscience, coupled with the development of new methods that could be applied in studies of postmortem brain tissue, created opportunities for novel and powerful probings into the pathobiology of psychiatric disorders. Indeed, based on the current demands for access to postmortem human brain specimens, the interest in these types of investigations of psychiatric disorders has never been greater. 
The direct study of the postmortem human brain provides several essential elements in the study of psychiatric disorders that are not currently, and that are not likely to be in the foreseeable future, available through other approaches. For example, although in vivo neuroimaging studies provide insights into critical areas (e.g., regional patterns of functional activation, dynamic changes in ligand receptor binding, and volumetric changes across time) that cannot be assessed in postmortem material, only postmortem studies provide the resolution required for the characterization of psychiatric disorders at the level of populations of neurons and the specific neural circuits that they form. That is, at present, disease-related alterations in local and distributed neural circuits, and at the cellular, synaptic and molecular levels, can only be detected through the direct study of brain tissue. Similarly, as susceptibility genes are identified for psychiatric disorders, postmortem tissue studies will provide an indispensable means for determining how those genetic liabilities are converted into altered expression of gene products. Furthermore, application of the recent powerful advances in genomics, and the anticipated opportunities in proteomics, to the study of psychiatric disorders requires the availability of postmortem brain specimens. For example, cDNA microarray studies of postmortem prefrontal cortex from subjects with schizophrenia have revealed abnormalities in the expression of multiple transcripts encoding proteins involved in specific domains of neural function (Mirnics et al. 2000, 2001a; Hakak et al. 2001; Vawter et al. 2001), providing a basis for the formulation of novel hypotheses regarding the pathobiology and the clinical heterogeneity of the disorder (Mirnics et al. 2001b). These types of studies now offer the promise of advances on a number of fronts, from the molecular phenotyping of psychiatric disorders into more homogeneous categories, to the generation of new candidates for susceptibility or protective genes, to the delineation of pathophysiological cascades, and to the identification of novel targets for therapeutic interventions. Thus, studies of the postmortem human brain represent a critical and complementary approach to in vivo studies, as well as an essential interface between clinical investigations and studies in animal models.

The quality of the best postmortem studies of human psychiatric disorders has, without question, improved substantially over the past two decades, whether one judges quality by larger sample sizes, by the inclusion of better and more appropriate controls, or by the increased rigor of experimental design and analysis. The practical outcome of these improvements, and of the increase in the number of such studies, has been the replication of findings across independent investigations. For example, three published studies have found evidence of reduced numbers of glial cells in regions of the frontal cortex in subjects with depression (Ongur et al. 1998; Rajkowska et al. 1999; Cotter et al. 2001). Within the field of schizophrenia research, three published studies have reported a similar $\sim 30 \%$ decrement in the number of neurons in the mediodorsal thalamic nucleus (Pakkenberg 1990; Popken et al. 2000; Young et al. 2000), two groups have found a reduction of somal size in deep layer 3 pyramidal neurons in prefrontal area 9 (Rajkowska et al. 1998; Pierri et al. 2001) and three independent research groups have found decreased expression of the mRNA for the 67 kilodalton isoform of glutamic acid decarboxylase $\left(\mathrm{GAD}_{67}\right)$, the synthesizing enzyme for GABA, in the dorsal prefrontal cortex (Akbarian et al. 1995; Guidotti et al. 2000; Volk et al. 2000) of subjects with schizophrenia.

Consequently, the opportunity for studies of the postmortem human brain to exploit rapidly developing technologies in the service of advancing our knowledge of psychiatric disorders has never been greater. However, as in any area of scientific investigation, such studies must be conducted with an adequate awareness of their strengths and limitations, and with appropriate attention to the types of issues that can maximize the strengths and mitigate the limitations. In particular, realization of the anticipated insights into the pathogenesis and pathophysiology of psychiatric disorders offered by postmortem human brain studies depends upon continued improvements in the design, execution and interpretation of these studies. To quote an adage from sports philosophy, "If you're not getting better, you're getting worse." Because a number of excellent reviews have discussed many of the important methodological issues involved in postmortem studies of psychiatric disorders (Benes 1988; Casanova and Kleinman 1990; Harrison and Kleinman 2000), the purpose of this Perspective is to highlight some areas in the planning and conduct of postmortem studies that require particular attention and to offer suggestions for improvements in these areas. Specifically, the premise of this paper is that the quality, and consequently the information value, of a postmortem human study depends upon the extent to which it employs well-characterized brain specimens, a well-constructed experimental design, and well-controlled confounds.

\section{Well-characterized Brain Specimens}

The ideal postmortem human brain study would utilize only "pristine" specimens; that is, tissue specimens not confounded by any factors. Of course, this ideal cannot be achieved because all specimens are postmortem and thus, by definition, are confounded in some way. By analogy, if the living human brain represents a featurelength movie, then the postmortem brain is a single frame, whose appearance may have been altered to different degrees by a variety of factors. In order to obtain 
meaningful information from this single frame, one has to know both how it has been altered and where it fits into the full-length film. Thus, a critical starting point in the conduct of postmortem human studies is the systematic characterization of the subjects and tissue samples to be investigated.

\section{Clinical Diagnosis}

The study of psychiatric disorders, including postmortem investigations, continues to rest upon syndromal diagnoses based on clinical features. Thus, as in clinical investigations, the quality of a postmortem study depends upon the extent and accuracy of the knowledge that supports the presence of the disorder under investigation. Of equal importance is the knowledge, obtained with a similar level of certainty, that the normal comparison subjects do not have a history of a psychiatric disorder. Just as structured interviews, with documented reliability, have replaced chart diagnoses or self-report in clinical studies, similar diagnostic rigor is an essential element in the characterization of subjects for postmortem studies. Structured, antemortem diagnostic assessments have been used quite effectively in some postmortem studies of schizophrenia (Arnold et al. 1998; Sokolov et al. 2000). The advanced age, chronic hospitalization status and substantial cognitive impairment of most subjects included in such studies suggest that they represent a unique and important subgroup of subjects with schizophrenia. However, the distinctive features of such subjects constrains the extent to which findings from these studies can be generalized to other forms or earlier stages of the illness. Indeed, studies of elderly, chronically hospitalized subjects with schizophrenia have yielded some findings such as increased levels of synaptophysin protein in the prefrontal cortex (Gabriel et al. 1997), that appear to be in the opposite direction to the decreased levels of this protein observed in studies of younger subjects with the disorder (Perrone-Bizzozero et al. 1996; Glantz and Lewis 1997; Karson et al. 1999).

For most postmortem studies, especially of individuals in the early to mid-stages of a psychiatric disorder, who are most likely to die by suicide or by an accident or illness not apparently related to the primary disease process, such prospective, antemortem assessments are not possible. The most direct route of obtaining clinical information on these subjects, chart review, is limited in several respects. First, chart data are by definition anecdotal, the amount and detail being dependent upon the clinicians recording the data and the extent to which the full chart history is actually available for review. Second, chart review is limited to subjects who have been seen by the medical profession. However, medical records can be a valuable source of information in concert with "psychological autopsies" which involve structured interviews of surviving relatives and friends and directed questioning of health care providers. Consequently, the use of psychological autopsies represents the most effective approach at present for the study of non-elderly, non-institutionalized subjects with psychiatric disorders and comparison subjects. The value of this approach has been demonstrated by Kelly and Mann (1996) who found that comparison of research diagnoses made solely by psychological autopsy with diagnoses made antemortem by a clinician generated a kappa coefficient of 0.85 for Axis I diagnoses, a degree of agreement similar to that found in reliability studies involving direct interviews with patients.

Presumed "normal" comparison subjects should also be subjected to the same diagnostic procedures used for subjects with psychiatric disorders. In our experience with subjects identified through a coroner's office, diagnosable psychiatric disorders, especially of substance abuse or dependence, are not uncommon in subjects for whom the initially available clinical records indicated no history of psychiatric illness.

Whether diagnostic information is obtained from antemortem assessments or psychological autopsies, the formulation of these data into consensus DSM-IV diagnoses may be best accomplished by an independent panel of experienced research clinicians. Such an approach circumvents the potential biases of investigators who will be studying the postmortem tissue and provides additional parallels to the approaches that produce high diagnostic reliability in clinical studies. For example, in The University of Pittsburgh's Center for the Neuroscience of Mental Disorders, the same team of research clinicians render consensus diagnoses for both the living subjects enrolled in clinical studies and the deceased subjects utilized in tissue-based studies.

Suggestions for Improvements. In essence, the same emphasis on the systematic acquisition of clinical data, and reliability and consensus in the diagnostic process, that characterize state-of-the-art clinical studies can be applied in postmortem investigations. Toward this end, an NIMH-sponsored workshop on approaches to diagnosis in postmortem studies may be of benefit. Such a workshop, including both individuals responsible for diagnostic issues in postmortem studies and established experts in clinical diagnosis and psychometric measures, could address issues such as the most appropriate instruments to use in data acquisition and the development of new methods for assessing and improving diagnostic reliability.

The information content of postmortem investigations may also be enhanced by examining the relations between the biological outcome measures of such studies and psychopathological features of the study subjects. For example, in schizophrenia research, the association between the magnitude of alterations in components of dorsal prefrontal cortical circuitry and severity of cog- 
nitive dysfunction might be particularly informative. However, since direct cognitive assessments cannot be made in a prospective fashion in most studies, the development of standardized instruments designed to quantify surrogate markers of cognitive function is needed.

Future postmortem studies may explore the neurobiology of intermediate or endophenotypes of psychiatric disorders; that is, traits found in both affected individuals and their unaffected relatives (Gottesman and Shields 1982). In addition to the advantages of this approach demonstrated in clinical investigations (Freedman et al. 1997; Egan et al. 2001), the study of deceased unaffected relatives who have passed through the age of risk permits the assessment of neurobiological measures of interest in the absence of the effects of drug treatment or other consequences of the illness. However, bringing such an approach to fruition depends both upon the identification of endophenotypes that are assessable in potential study subjects and the recovery of postmortem brain specimens from unaffected relatives. In our experience, the parents of deceased individuals with a major psychiatric disorder frequently express interest in becoming brain donors upon their own death. Thus, in these individuals, prospective antemortem assessments may be coupled with the recovery of brain tissue within a reasonable time frame. Postmortem studies of such parent-child dyads (or triads) might be especially informative, but the acquisition of adequate sample sizes would clearly require a substantial and long-term investment.

\section{Neuropathological Assessment}

The psychiatric disorders that are the focus of most postmortem studies (e.g., bipolar disorder, major depression and schizophrenia) do not have any known features that can be detected by standard neuropathological assessments. However, these assessments are still obviously important both to "rule out" other diseases that may mimic the clinical features of psychiatric disorders and to characterize the presence of brain abnormalities that might affect study design or interpretation. Clearly, the types and incidence of neuropathological abnormalities are likely to differ across psychiatric subjects as a function of a variety of factors (e.g., age, cause of death, community or hospital-based death, etc.). However, not every abnormality detected on neuropathological exam necessarily represents an exclusion condition, an important consideration given the importance (discussed below) of increasing the sample sizes employed in postmortem studies. Thus, the goal of neuropathological inquiry is to ascertain the presence of all abnormalities so that the potential impact of each can be considered in the design of each study. At the same time, it is important to minimize the impact that neuropathological assessment has on the availability of brain tissue for subsequent studies.

Suggestions for Improvements. Although most published postmortem studies provide some information regarding neuropathological examination, the basis for the information provided is not often apparent. Involvement of an interested neuropathologist in the brain collection process makes it possible to approach the assessment on an individualized basis, guided by the clinical record and gross tissue inspection in order to obtain both standard tissue samples and additional ones as appropriate, with minimal disruption of the preferred tissue blocking regimen. In addition, the ongoing participation of the neuropathologist facilitates the review of sections from additional tissue blocks as they are cut for use in studies.

\section{Antemortem Factors}

The integrity of the morphology and biochemical content of brain structures may be affected by factors that are operative prior to death. Alterations in acid-base balance, blood oxygenation and brain perfusion that may occur during the agonal state can have complex effects on the dependent measures of interest in postmortem studies. Although these factors may, in general, be less of a concern in subjects who died suddenly, outside of a medical care setting (e.g., due to automobile accidents, myocardial infarctions or arrhythmias) than in individuals who died following a prolonged illness that involved medical interventions (e.g., due to pneumonia or cancer), cause and manner of death do not readily lend themselves to quantification or to comparisons across subject groups. However, the severity of agonal state has been reported to be inversely proportional to postmortem brain $\mathrm{pH}$, which appears to be relatively uniform across the brain (Harrison et al. 1995). In contrast, brain $\mathrm{pH}$ does not appear to change as a function of postmortem interval (Ravid et al. 1992; Kingsbury et al. 1995).

Although enzyme activity measures appear to be affected by brain pH (Perry et al. 1982; Yates et al. 1990), immunoreactivity of brain proteins and ligand binding to receptors do not appear to differ substantially as a function of brain $\mathrm{pH}$ (Harrison et al. 1995). However, tissue levels of many species of mRNAs are directly related to brain pH (Harrison et al. 1995; Kingsbury et al. 1995). Based on observations that sudden deaths are associated with brain $\mathrm{pH}$ of $\sim 6.8$ and prolonged agonal states may produce brain $\mathrm{pH}$ of $<6.0$, Harrison and Kleinman (2000) have recommended that all brains be screened for $\mathrm{pH}$ and those with values $<6.1$ be excluded from study.

In addition to manner and cause of death, the possible effects of season and time of death may also need to be considered. Clearly, seasonal and circadian variations in- 
fluence measures of hypothalamic function, whether assessed pre- or postmortem (Ravid et al. 1992).

Suggestions for Improvements. Although cause and/ or manner of death are frequently reported in postmortem studies of psychiatric disorders, with the exception of suicide, this information is rarely utilized in study design or interpretation. Binary classification schemes, such as sudden versus prolonged death, or as following versus not following medical intervention, would make information regarding cause of death more tractable. However, it is not clear that such schemes, even if reliable, would meaningfully improve the characterization of the brain specimens under study.

In terms of markers of agonal state events, the detection on neuropathological exam of cellular changes attributable to hypoxia may serve as a grounds for excluding a brain specimen from study. The available data also suggest that postmortem brain $\mathrm{pH}$ is both informative about certain types of antemortem factors and provides a continuous variable that can be utilized as a covariate in statistical models. However, the value of brain $\mathrm{pH}$ measures may be enhanced through a better understanding of the factors that affect these measures. For example, studies in rodents in which cerebral hypoxia has been induced (and reversed) in appropriate experimental paradigms may be of use. Tissue from such studies would also permit a more controlled assessment of the relationship of brain $\mathrm{pH}$ to dependent measures of interest than is possible in comparisons across human brains.

It may also be informative to examine the possible influence of time and season of death, as well as sleep-wake status at time of death, and to report this information for each subject. Clearly, controlled studies in appropriate species of animals are needed to address the possible impact of these factors in postmortem human studies.

\section{Postmortem Factors}

In addition to clinical and neuropathological diagnoses, the assessment of postmortem interval (PMI), the elapsed time between death and the freezing or immersion of brain tissue in fixative, is a frequently utilized measure of the quality of postmortem brain specimens. Although it is reasonably assumed that the shorter the PMI, the better the quality of the tissue, this view does not take into consideration a number of relevant factors. For example, the effect of PMI on a given dependent variable may be complex. Thus, the absence of a significant linear relationship across study subjects between PMI and a biological measure does not exclude an influence of postmortem factors. Among other explanations, the absence of this association could mean that the biological measure had already changed substantially from the in vivo state at the earliest PMI examined.
Indeed, a limited number of controlled experimental investigations of PMI on morphometric measures or the detectability of certain molecules demonstrate the nonlinear nature of postmortem effects. For example, in a study of different PMIs produced in adjacent blocks of neocortex from the same monkeys, the somal size of a specific class of neurons was directly associated with PMI across a range of $30 \mathrm{~min}$ to $12 \mathrm{~h}$, but did not change between 12 and $48 \mathrm{~h}$ (Hayes et al. 1991). The tissue concentrations of individual members of a family of proteins may also change in different ways as a function of PMI. Figure 1 compares the tissue concentrations of three prosomatostatin-derived peptides in biopsy samples of monkey prefrontal cortex that were frozen at different time intervals after removal, creating different "PMIs" (Hayes et al. 1991). Note that the concentration of one peptide, somatostatin-28, declined to $10-20 \%$ of baseline levels at the 10 min PMI, whereas the concentrations of two other peptides, somatostatin-14 and somatostatin-28 $8_{1-12}$, actually increased during the same time interval, presumably as a consequence of the cleaving of somatostatin-28. However, by $12 \mathrm{~h}$ PMI, each of these peptides showed a different relative tissue concentration compared with its baseline level. That is, somatostatin-28 levels remained stable at about $20 \%$ of baseline, somatostatin-14 levels were approximately $60 \%$ of baseline, and somatostatin-28 $8_{1-12}$ levels had returned to baseline levels. Similar complex, PMI-related effects have also been observed for different isoforms of the same protein (Lewis et al. 1993, 1994).

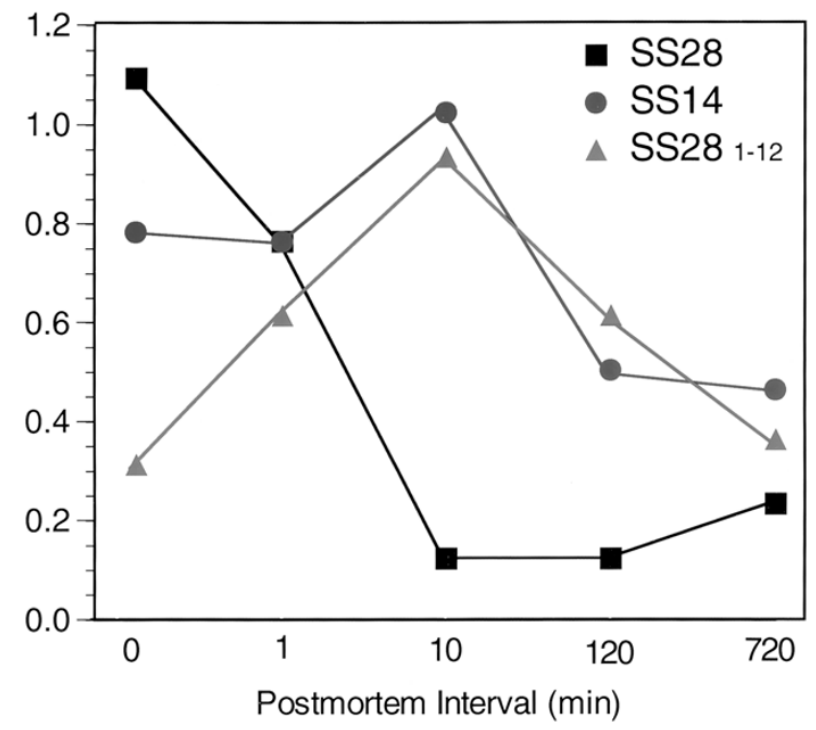

Figure 1. Tissue concentrations (ng/mg protein) of three pro-somatostatin-derived peptides in biopsied tissue from area 9 of monkey prefrontal cortex following different "postmortem intervals." Note the complex and distinctive patterns of change in the concentrations of somatostatin-28 (SS28), somatostatin-14 (SS14) and somatostatin-281-12. Data adapted from Hayes et al. (1991). 
PMI may also affect immunoreactivity for the same protein in a non-uniform fashion across brain structures, and even across layers within the same region of the cerebral cortex. For example, an antibody directed against nonphosphorylated epitopes of neurofilament proteins (NFP) identifies a subpopulation of neurons in the human entorhinal cortex with distinctive regional and laminar patterns of distribution (Beall and Lewis 1992). In the intermediate subdivision of the human entorhinal cortex, intensely immunoreactive NFP-positive neurons are present in layers 2 and 5 (Figure 2, panel A). Interestingly, in a pilot study of schizophrenia, NFP-immunoreactive neurons were absent in layer 2, but were clearly detectable in layer 5 (Figure 2, panel B), suggesting the possibility of laminar- and neuron-specific disturbance in this illness (Lewis and Akil 1997). However, in adjacent thin tissue blocks of monkey entorhinal cortex fixed at varying PMIs, NFP-immunoreactive neurons were clearly present in both layers 2 and 5 following a $30 \mathrm{~min}$ PMI (Figure 2, panel C), but in layer 2 the overall intensity of immunoreactivity was substantially reduced at $12 \mathrm{~h}$ PMI and was undetectable following a $24 \mathrm{~h}$ PMI (Figure 2, panel D). In contrast, layer 5 neurons remained clearly immunoreactive up to a $48 \mathrm{~h}$ PMI. These findings demonstrate that PMI effects on the same protein can differ across cortical layers within the same brain region, and they illustrate how such complex effects could be misinterpreted as the result of a disease-specific process.

In addition to PMI, the type and length of storage of brain specimens represents another postmortem factor that may affect the outcome of a study. For example, tissue preservation by continued immersion in formalin or another fixative for varying lengths of time may result in different degrees of tissue shrinkage that may be difficult to assess or account for, depending upon the type of study to be conducted. Prolonged fixation also substantially reduces the detectability of proteins by immunocytochemistry, although antigen-retrieval techniques, such as exposure of tissue specimens to elevated temperature or microwave irradiation, have produced remarkable recovery of immunoreactivity. However, limited data exist regarding whether these techniques actually restore immunoreactivity to the level detectable with an ideal degree of fixation and whether the recovery of immunoreactivity is actually comparable across specimens with different lengths of fixation. The available data do suggest that immunoreactivity is not altered in tissue fixed for a standard period time and then stored in cryoprotectant at $-30^{\circ} \mathrm{C}$ (Erickson et al. 1998). Finally, freezer storage time for fresh-frozen tissue has been reported to be negatively correlated with tissue levels of some, but not all, mRNA transcripts (Burke et al. 1991; Harrison et al. 1995).

Suggestions for Improvements. Each of the examples of PMI effects presented above may represent more the
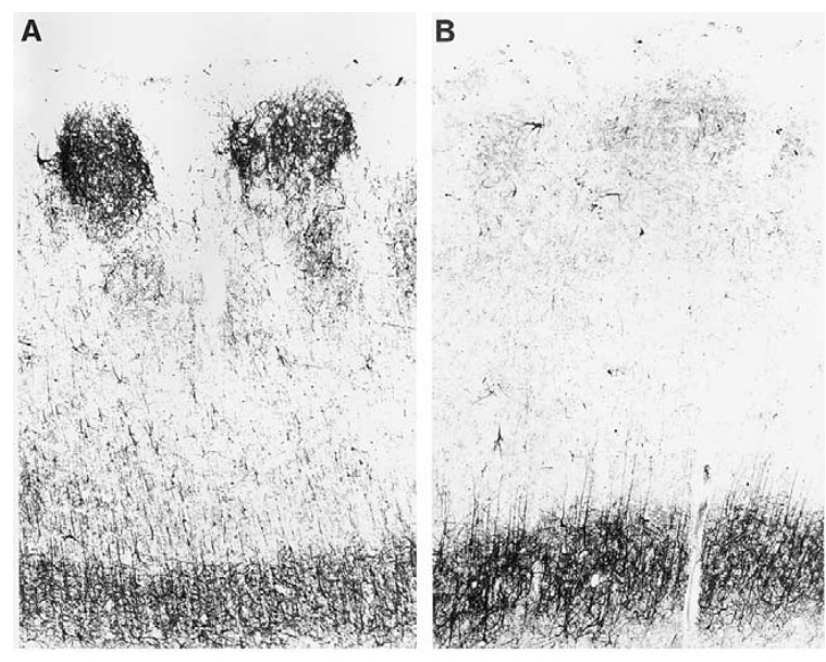

C

D
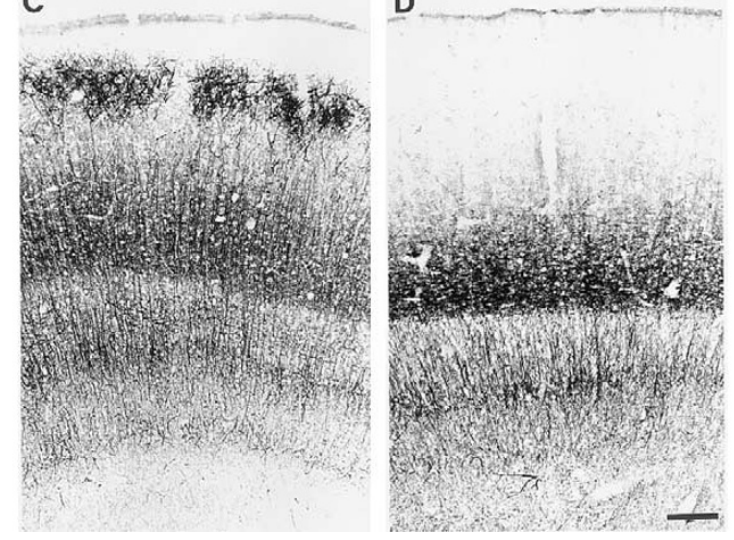

Figure 2. Brightfield photomicrographs comparing nonphosphorylated neurofilament protein (NFP) immunoreactivity in the intermediate subdivision (EI) of human entorhinal cortex from a normal control subject (A) and a subject with schizophrenia (B). Note the absence of NFP immunoreactivity in layer II clusters of neurons in the subject with schizophrenia, despite the presence of these neurons on an adjacent Nissl-stained section (not shown) and the presence of intensely immunoreactive neurons and dendrites in the deep cortical layers, similar to that of the normal control subject. However, the decreased NFP immunoreactivity in layer II of panel A appears to represent a postmortem effect rather than a schizophrenia-related change as indicated by the result of experiments summarized in panels $\mathbf{C}$ and $\mathbf{D}$. These panels show NFP immunoreactivity in sections from adjacent tissue blocks, containing the same subdivision of monkey entorhinal cortex, that were processed following postmortem intervals of 0.5 (C) and 24 (D) hours. Note that the longer PMI (D) results in a loss of NFP immunoreactivity that is restricted to layer II. Scale bar $=150 \mu \mathrm{m}$ and applies to all panels. Figure reprinted from Lewis and Akil (1997).

exception than the rule, and some (i.e., postmortem processing within the family of prosomatostatin-derived proteins) may represent extreme cases. Indeed, a number of studies suggest that other classes of molecules, 
such as mRNAs, may be extremely stable following death. These examples do, however, illustrate the potentially complex and substantial impact of postmortem effects, and thus they demonstrate the need to systematically assess and control for such effects in designing postmortem studies.

Clearly, the more information that is available about the PMI, the better the characterization of the tissue specimens. In addition to accurately determining the interval between death and tissue preservation, some investigators have suggested that the intervals between several time points (i.e., death, refrigeration of body, brain removal, and brain preservation) may be more informative (W. Bunney, personal communication). However, knowing how to include such information in experimental design and statistical analyses requires further investigation. The identification of a reliable, quantitative surrogate measure that would provide a global estimate of the effects of processes operating after death would be very helpful. Such a measure would provide a means of assessing the relative influence of postmortem factors within and across subject groups in a study and for making comparisons across studies. Perhaps of greater importance, and certainly more immediately feasible, is the experimental assessment in an appropriate animal model system of the impact of PMI on the specific dependent measure under study. By providing a priori knowledge of the potential confound due to PMI, this information could be used to determine which subjects are appropriate for study, and whether the proposed study can actually produce interpretable results in the available subjects. Toward this end, the availability of standardized brain tissue samples from nonhuman primates in which the only difference across samples is length of PMI would permit a more uniform and reliable assessment of PMI effects.

What length of PMI is acceptable? Although it is tempting to pick an arbitrary cut-off value such as $24 \mathrm{~h}$, the available data indicate that the range of acceptable PMIs clearly depends upon the measurement being made. Furthermore, PMI cannot be considered in isolation from antemortem or other factors. For example, in a study of gene expression, it may be that the brain of an individual who died of a prolonged illness with progressive hypoxia, but with a PMI of less than $5 \mathrm{~h}$, is less suitable than the brain of an individual who died suddenly of a cardiac arrhythmia but with a PMI of $24 \mathrm{~h}$.

\section{Toxicology}

Interpretation of findings from a postmortem study requires a means to determine whether the observation is influenced by the pharmacological treatment of the illness of interest or by other agents that may have effects on the central nervous system. This determination depends upon knowledge of which medications the sub- ject received, for how long and at what dosage. However, such detailed information is difficult to obtain, frequently incomplete, confounded by compliance issues and usually not available to the same degree across subjects (with the possible exception of individuals who have been chronically hospitalized). Even when such information is available, it is difficult to know how to make comparisons across subjects: for example, is the relevant measure dose at time of death, total life time dose, etc.? Finally, a substantial percentage of subjects have received more than one psychotropic medication, and the extent to which drug interactions confound the picture is difficult to assess. In addition to prescribed medications, the potential influence of other commonly used psychotropic agents, such as alcohol, caffeine and nicotine, need to be considered, with the relevance of each of these variables dependent upon the nature of the study to be conducted.

Suggestions for Improvements. Assessment of the potential impact of psychotropic agents in postmortem studies would be enhanced by improved means to detect the presence of such agents in study subjects. At present, this assessment is usually limited to assays of blood or other bodily fluids and is not uniformly available to all investigators who acquire postmortem brain specimens. The development of a centralized national core resource for conducting these assays would substantially improve the quality and uniformity of postmortem studies. In addition to conducting drug assays on serum or other fluids submitted by investigators in the field, this core could adapt or develop methods for measuring drug concentrations in brain tissue and hair. Such measures would, respectively, provide data that may be more biologically relevant and that reflect exposure over the several months preceding death.

\section{Well-constructed Studies}

As in other areas of investigation, the design of postmortem studies requires attention to a number of central issues that may affect outcome and interpretation. Perhaps most challenging and difficult for the investigator is the decision of whether the available postmortem tissue specimens can support the proposed studies. That is, given the question to be addressed, are the available tissue specimens appropriate in terms of the types of variables outlined in the preceding section? Stated in another way, the investigator must determine if the proposed study, independent of the interest or importance of the question to be addressed, is actually worth conducting. If the available tissue specimens are poorly characterized, or if they are suboptimal in some respect (e.g., $\mathrm{pH}$ values <6.1), then the investigator (and possibly manuscript referees and editors) must decide whether potentially flawed or misleading data are better than no data at all. Although a fundamental ele- 
ment in the success of the scientific endeavor is its selfcorrecting nature, the effectiveness and extent to which this principle operates is dependent, in part, upon the number of investigators in the field and the rate at which studies are completed. Although certainly the number of postmortem studies of psychiatric disorders has increased in recent years, relatively few questions have been addressed with a sufficient number of studies to obtain definitive answers. However, any conceptually compelling finding from a postmortem study may be viewed by the field as a critical and foundational truth. Consequently, the great risk of poorly designed or controlled postmortem studies is that the resultant errant finding may persist untested or unchallenged for an extended period of time. Hence, although true in any area of science, given the relatively small number of postmortem studies, and the length of time that it takes to conduct them, the importance of well-conceptualized and designed postmortem studies of psychiatric disorders cannot be overemphasized.

Suggestions for Improvements. Statistical methods can be used to correct for differences in demographic (e.g., age, sex, etc.) or other (e.g., brain pH, PMI, tissue storage time, etc.) variables across subject groups. However, the matching of individual pairs (or triads or tetrads) of subjects from each group as closely as possible on these variables has several advantages. First, matching helps ensure that both the means and standard deviations for a given variable will be comparable across subject groups, further enhancing the utility of statistical corrections for these factors. Second, for assays in which tissue samples from all subjects cannot be processed together, matching of subjects across diagnostic groups, and always processing tissue from matched subjects together, helps reduce the potentially confounding influence of inter-assay variation. Third, matching subjects makes it possible to compare the results of different statistical models, in which subject pair and/or one or more of the matching variables are entered as blocking variables or covariates (for example, see Pierri et al. 2001). Comparable results across models provides additional support for the finding of interest. Clearly, however, this strategy requires the availability of a large number of normal subjects (or subjects in other diagnostic groups) in order to be able to select comparison subjects that are adequately matched on the relevant variables.

Although some issues about the application of stereological principles to studies of the brain have generated substantial debate (Guillery and Herrup 1997; West 1999; Benes and Lange 2001), the critical importance of systematic, uniformly random sampling to obtain valid estimates of the density or absolute number of objects in a region is not controversial. By extrapolation, one can reasonably conclude that estimates of the tissue concentration of a given molecule would also benefit from a similar approach to selecting the bits of tissue to be sampled. However, limitations in tissue specimens can present problems in this area (e.g., the use of a single tissue section per subject from a heterogenous brain region). Although using a larger sample of subjects may help mitigate the problem, the clear solution for advancing the field is to increase the availability of resources so that an appropriate number of brains is adequately sampled in all studies.

\section{Well-controlled Confounds}

Inherent in the use of postmortem tissue to study psychiatric illness is the challenge of disentangling the nature of the primary disease process (the usual focus of the study) from: (1) the consequences of having had the illness for years or possibly decades; (2) the influence of other disorders, such as substance abuse or suicide, that may be comorbid with the illness of interest; and (3) the impact of pharmacological agents used to manage the illness.

Primary versus Secondary Factors. With the exception of some cases of suicide, most individuals with psychiatric disorders who become available for a postmortem study have had the illness for some period of time. However, some inferences regarding whether an observed alteration in the dependent measure of interest is a consequence of having had the illness may be drawn from assessments of the relationship between the magnitude of change in the dependent measure and the length of illness (with appropriate corrections for age at time of death). Within the field of schizophrenia research, the majority of such analyses have failed to find a significant association. Whether these observations represent true negatives, or the blunted nature of the assessment (i.e., because the measure of interest, while a consequence of the illness, does not change linearly with length of illness), remains to be seen. However, the inference can be strengthened by comparing related measures between postmortem and in vivo imaging studies. For example, the likelihood that the reductions in volume and neuron number observed in the postmortem mediodorsal thalamus of subjects with schizophrenia (Pakkenberg 1990; Popken et al. 2000; Young et al. 2000) are related to the primary disease process is supported by the reported reductions in total thalamic volume (Buchsbaum et al. 1996; Gur et al. 1998; Gilbert et al. 2001) and in the size of the mediodorsal nucleus (Byne et al. 2001) in first-onset, never-medicated subjects with schizophrenia. A third strategy that has been less frequently utilized is the study of unaffected relatives of ill subjects. Although confounds of age have to be considered, these individuals may be both the most practical and useful to study postmortem because they have passed through the age of risk and could be potentially assessed antemortem. Although each of these approaches has definite limita- 
tions in addressing the question of whether a given postmortem finding reflects the disease process or its consequence, a convergence of findings across the three approaches may provide a compelling conclusion.

Influence of Comorbid Conditions. The necessity of dissecting the influence of the primary illness from that of comorbid conditions, such as alcohol and other substance use disorders, is also an important issue that affects the design of postmortem studies. Indeed, because of the high lifetime incidence of substance-related diagnoses in individuals with schizophrenia, which may approach 50\% (Dixon et al. 1991), and the limited sample of postmortem samples available for study, it is difficult to advocate a strategy of excluding all subjects with any history of a substance use disorder. Alternatively, one can look for compatible findings from several types of analyses. For example, the comparison of individuals with schizophrenia who have the comorbid condition with those who do not may be informative, although the resulting reduction in sample size runs the risk of diminished power and false negative findings. However, if both subgroups show a similar difference from normal control subjects, then the provisional conclusion of a lack of influence of the comorbid condition may be reasonable. This conclusion may be strengthened by the study of individuals who have the comorbid condition without the primary disease of interest. Two independent studies of gene expression illustrate the utility of this combined approach. For example, expression of the mRNA for N-ethylmaleimide sensitive factor was consistently decreased in the prefrontal cortex of subjects with schizophrenia, independent of whether they had history of alcoholism (Mirnics et al. 2000). In contrast, the expression of this mRNA was increased in subjects with alcoholism who did not suffer from schizophrenia (Lewohl et al. 2000).

Impact of Pharmacological Interventions. Since an adequate sample of postmortem brain specimens from never-medicated subjects with a given psychiatric disorder is unlikely to ever be available, several less direct approaches must be used to address the influence of psychotropic medications. These approaches include: (1) the comparison of data from subjects who were on or off the medications of interest at the time of death; (2) the examination of subjects with other disorders who were treated with the same medications; and (3) the use of animal models that mimic the clinical treatment of the disorder under investigation. Certainly, the first two approaches have obvious, and difficult to control, potential confounds. Long-term exposure to psychotropic medications, as is typical in the treatment of most psychiatric disorders, may have effects on brain morphology, neurochemistry or gene expression that persist for a substantial period of time after the drug is discontinued. In addition, even though the same agents are utilized, the pharmacological treatment of other dis- orders may be different than that of the disease of interest. For example, compared with schizophrenia, the use of antipsychotic medications for other psychotic disorders tends to be more intermittent or for a shorter duration of time. In the case of animal models of drug effects, studies in rodents frequently involve dosage and/ or length of treatment parameters that do not reflect the human treatment condition. These limitations can be overcome through studies in nonhuman primates that involve extended periods of treatment with medication dosing regimens that produce serum drug levels demonstrated to be therapeutic in humans. However, the potential problem of species differences, and the possibility that the medications of interest may have different effects on the brain of an individual with a psychiatric illness than on the normal brain must be kept in mind. Despite the limitations of each of these three approaches individually, convergent findings across approaches should lead to reasonable conclusions about the extent to which psychotropic medications account for brain differences between affected and normal control subjects.

This three-fold strategy has been used to assess the potential relationship of antipsychotic drug treatment to the reduced density of GABA transporter (GAT)-1labeled axon terminals (termed "cartridges") of chandelier neurons observed in the prefrontal cortex of subjects with schizophrenia. First, GAT-1 cartridge density was decreased in subjects with schizophrenia, but not in subjects with other psychiatric disorders treated with antipsychotic medications (Woo et al. 1998). Second, the density of labeled cartridges was actually greater in schizophrenic subjects who were off antipsychotic medications at the time of death compared with those on medications, although both groups showed reduced levels compared with normal controls (Pierri et al. 1999). Third, compared with matched control animals, the density of GAT-1-positive cartridges was elevated in monkeys treated for one year with haloperidol at serum levels maintained in the range shown to be therapeutic in humans (Pierri et al. 1999). Thus, the convergence of these findings suggest not only that antipsychotic medications are unlikely to be the cause of decreased GAT-1-immunoreactive cartridge density in schizophrenia, but also that the pathophysiology of schizophrenia may actually be associated with more marked reductions in GAT-1-immunoreactive cartridge density than those observed in postmortem studies.

\section{CONCLUSIONS}

As summarized at the beginning of this Perspective, investigation of the postmortem human brain is an essential component of the current effort to unravel the complex pathoneurobiology of psychiatric disorders. 
The success of this endeavor depends upon continued improvements in both the quality and quantity of such studies. In addition to the specific suggestions delineated above, increasing the number of postmortem brain specimens available for study is central to the success of this arm of the scientific assault on psychiatric illness. Although the relative merits of national versus local brain banks have been debated, the need for more brain specimens suggests that this may not be an "eitheror" question. Furthermore, the decreased number of hospital-based autopsies, as well as the additional confounds that may be associated with in-hospital deaths, indicate that efforts to increase alliances with coroner or medical examiner offices and with programs that recover organs and tissues for transplantation are needed.

Even with increased acquisition of well-characterized brain specimens, the confounds inherent to postmortem tissue research necessitate the use of convergent approaches to explicate the possible influence of these confounds. In this regard, both the design and interpretation of human postmortem studies may be improved by conducting them within the context of parallel animal studies of the same systems of interest in which potential confounds are assessed in a systematic fashion.

In summary, informative postmortem studies require well-characterized tissue specimens in which: (1) the potential confounds are understood in advance and are addressed through the design of the study; and (2) appropriate control experiments are conducted to assess the possible impact of these confounds on the dependent measures of interest. Continued efforts to improve our abilities to meet these requirements will help insure that postmortem studies provide the essential insights into psychiatric disorders that can only be achieved through the direct investigation of the human brain.

\section{ACKNOWLEDGMENTS}

Although the author assumes full responsibility for the content of this article, he wishes to acknowledge and thank the participants in an NIMH-sponsored workshop on "Postmortem Brain Analysis" (September 18-19, 2000; Bethesda, MD) for the open and valuable discussions that influenced his thoughts on this subject. Cited studies conducted by the author were supported by NIH grants MH45156, MH00519 and MH43784.

\section{REFERENCES}

Akbarian S, Kim JJ, Potkin SG, Hagman JO, Tafazzoli A, Bunney WE Jr, Jones EG (1995): Gene expression for glutamic acid decarboxylase is reduced without loss of neurons in prefrontal cortex of schizophrenics. Arch Gen Psychiatry 52:258-266
Arnold SE, Trojanowski JQ, Gur RE, Blackwell P, Han L-Y, Choi C (1998): Absence of neurodegeneration and neural injury in the cerebral cortex in a sample of elderly patients with schizophrenia. Arch Gen Psychiatry 55: 225-232

Beall MJ, Lewis DA (1992): Heterogeneity of layer II neurons in human entorhinal cortex. J Comp Neurol 321:241-266

Benes FM (1988): Post mortem structural analyses of schizophrenic brain: Study designs and the interpretation of data. Psychiat Dev 3:213-226

Benes FM, Lange N (2001): Two-dimensional versus threedimensional cell counting: A practical perspective. Trends Neurosci 24:11-17

Buchsbaum MS, Someya T, Teng CY, Abel L, Chin S, Najafi A, Haier RJ, Wu J, Bunney WE Jr (1996): PET and MRI of the thalamus in never-medicated patients with schizophrenia. Am J Psychiatry 153:191-199

Burke WJ, O'Malley KL, Chung HD, Harmon SK, Miller JP, Berg L (1991): Effect of pre- and postmortem variables on specific mRNA levels in human brain. Mol Brain Res 11:37-41

Byne W, Buchsbaum MS, Kemether E, Hazlett EA, Shinwari A, Mitropoulou V, Siever LJ (2001): Magnetic resonance imaging of the thalamic mediodorsal nucleus and pulvinar in schizophrenia and schizotypal personality disorder. Arch Gen Psychiatry 58:133-140

Casanova MF, Kleinman JE (1990): The neuropathology of schizophrenia: A critical assessment of research methodologies. Biol Psychiatry 27:353-362

Cotter D, Mackay D, Landau S, Kerwin R, Everall I (2001): Reduced glial cell density and neuronal size in anterior cingulate cortex in major depressive disorder. Arch Gen Psychiatry 58:545-553

Dixon L, Haas G, Weiden PJ, Sweeney J, Frances AJ (1991): Drug abuse in schizophrenic patients: Clinical correlates and reasons for use. Am J Psychiatry 148:224-230

Egan MF, Goldberg TE, Kolachana BS, Callicott JH, Mazzanti CM, Straub RE, Goldman D, Weinberger DR (2001): Effect of COMT Val 108/158 Met genotype on frontal lobe function and risk for schizophrenia. Proc Natl Acad Sci USA 98:6917-6922

Erickson SL, Akil M, Levey AI, Lewis DA (1998): Postnatal development of tyrosine hydroxylase- and dopamine transporter-immunoreactive axons in monkey rostral entorhinal cortex. Cereb Cortex 8:415-427

Freedman R, Coon H, Myles-Worsley M, Orr-Urtreger A, Olincy A, Davis A, Polymeropoulos M, Holik J, Hopkins J, Hoff M, Rosenthal J, Waldo MC, Reimherr F, Wender P, Yaw J, Young DA, Breese CR, Adams C, Patterson D, Adler LE, Kruglyak L, Leonard S, Byerley W (1997): Linkage of a neurophysiological deficit in schizophrenia to a chromosome 15 locus. Proc Natl Acad Sci USA 94:587-592

Gabriel SM, Haroutunian V, Powchik P, Honer WG, Davidson M, Davies P, Davis KL (1997): Increased concentrations of presynaptic proteins in the cingulate cortex of subjects with schizophrenia. Arch Gen Psychiatry 54: 559-566

Gilbert AR, Rosenberg DR, Harenski K, Spencer S, Sweeney JA, Keshavan MS (2001): Thalamic volumes in patients with first-episode schizophrenia. Am J Psychiatry 158: 618-624 
Glantz LA, Lewis DA (1997): Reduction of synaptophysin immunoreactivity in the prefrontal cortex of subjects with schizophrenia: Regional and diagnostic specificity. Arch Gen Psychiatry 54:943-952

Gottesman II, Shields J (1982): Schizophrenia: The Epigenetic Puzzle. Cambridge, UK, Cambridge University Press

Guidotti A, Auta J, Davis JM, Gerevini VD, Dwivedi Y, Grayson DR, Impagnatiello F, Pandey G, Pesold C, Sharma R, Uzunov D, Costa E (2000): Decrease in reelin and glutamic acid decarboxylase67 (GAD67) expression in schizophrenia and bipolar disorder. Arch Gen Psychiatry 57:1061-1069

Guillery RW, Herrup K (1997): Quantification without pontification: Choosing a method for counting objects in sectioned tissues. J Comp Neurol 386:2-7

Gur RE, Maany V, Mozley PD, Swanson C, Bilker W, Gur RC (1998): Subcortical MRI volumes in neurolepticnaive and treated patients with schizophrenia. Am J Psychiatry 155:1711-1717

Hakak Y, Walker JR, Li C, Wong WH, Davis KL, Buxbaum JD, Haroutunian V, Fienberg AA (2001): Genome-wide expression analysis reveals dysregulation of myelination-related genes in chronic schizophrenia. Proc Natl Acad Sci USA 98:4746-4751

Harrison PJ, Heath PR, Eastwood SL, Burnet PWJ, McDonald B, Pearson RCA (1995): The relative importance of premortem acidosis and postmortem interval for human brain gene expression studies: Selective mRNA vulnerability and comparison with their encoded proteins. Neurosci Lett 200:151-154

Harrison PJ, Kleinman JE (2000): Methodological issues. In Harrison PJ, Roberts GW (eds), The Neuropathology of Schizophrenia. New York, Oxford University Press, pp 339-350

Hayes TL, Cameron JL, Fernstrom JD, Lewis DA (1991): A comparative analysis of the distribution of prosomatostatin-derived peptides in human and monkey neocortex. J Comp Neurol 303:584-599

Johnstone EC, Crow TJ, Frith CD, Husband J, Kreel L (1976): Cerebral ventricular size and cognitive impairment in chronic schizophrenia. Lancet 2:924-926

Karson CN, Mrak RE, Schluterman KO, Sturner WQ, Sheng JG, Griffin WST (1999): Alterations in synaptic proteins and their encoding mRNAs in prefrontal cortex in schizophenia: A possible neurochemical basis for 'hypofrontality'. Mol Psychiatry 4:39-45

Kelly TM, Mann JJ (1996): Validity of DSM-III-R diagnosis by psychological autopsy: A comparison with clinician antemortem diagnosis. Acta Psychiatr Scand 94:337-343

Kingsbury AE, Foster OJF, Nisbet A, Cairns N, Bray L, Eve DJ, Lees AJ, Marsden CD (1995): Tissue $\mathrm{pH}$ is an indicator of mRNA preservation in human post-mortem brain. Mol Brain Res 28:311-318

Lewis DA, Melchitzky DS, Haycock JW (1993): Four isoforms of tyrosine hydroxylase are expressed in human brain. Neuroscience 54:477-492

Lewis DA, Melchitzky DS, Haycock JW (1994): Expression and distribution of two isoforms of tyrosine hydroxylase in macaque monkey brain. Brain Res 656:1-13

Lewis DA, Akil M (1997): Cortical dopamine in schizophre- nia: Strategies for postmortem studies. J Psychiatry Res 31:175-195

Lewohl JM, Wang L, Miles MF, Zhang L, Dodd PR, Adron Harris R (2000): Gene expression in human alcoholism: Microarray analysis of frontal cortex. Alcohol Clin Exp Res 24:1873-1882

Mirnics K, Middleton FA, Marquez A, Lewis DA, Levitt P (2000): Molecular characterization of schizophrenia viewed by microarray analysis of gene expression in prefrontal cortex. Neuron 28:53-67

Mirnics K, Middleton FA, Stanwood GD, Lewis DA, Levitt P (2001a): Disease-specific changes in regulator of G-protein signaling 4 (RGS4) expression in schizophrenia. Mol Psychiatry 6:293-301

Mirnics K, Middleton FA, Lewis DA, Levitt P (2001b): Analysis of complex brain disorders with gene expression microarrays: Schizophrenia as a disease of the synapse. Trends Neurosci 24:479-486

Ongur D, Drevets WC, Price JL (1998): Glial reduction in the subgenual prefrontal cortex in mood disorders. Proc Natl Acad Sci USA 95:13290-13295

Pakkenberg B (1990): Pronounced reduction of total neuron number in mediodorsal thalamic nucleus and nucleus accumbens in schizophrenics. Arch Gen Psychiatry 47:1023-1028

Perrone-Bizzozero NI, Sower AC, Bird ED, Benowitz LI, Ivins KJ, Neve RL (1996): Levels of the growth-associated protein GAP-43 are selectively increased in association cortices in schizophrenia. Proc Natl Acad Sci USA 93:14182-14187

Perry EK, Perry RH, Tomlinson BE (1982): The influence of agonmortem human brain tissue. Neurosci Lett 29:303307

Pierri JN, Chaudry AS, Woo T-U, Lewis DA (1999): Alterations in chandelier neuron axon terminals in the prefrontal cortex of schizophrenic subjects. Am J Psychiatry 156:1709-1719

Pierri JN, Volk CLE, Auh S, Sampson A, Lewis DA (2001): Decreased somal size of deep layer 3 pyramidal neurons in the prefrontal cortex in subjects with schizophrenia. Arch Gen Psychiatry 58:466-473

Plum F (1972): Neuropathological findings. In Kety SS, Malthysse SM (eds), Prospects for Research on Schizophrenia. Cambridge, MA, MIT Press, pp 385-388

Popken GJ, Bunney WE Jr, Potkin SG, Jones EG (2000): Subnucleus-specific loss of neurons in medial thalamus of schizophrenics. Proc Natl Acad Sci 97:9276-9280

Rajkowska G, Selemon LD, Goldman-Rakic PS (1998): Neuronal and glial somal size in the prefrontal cortex: A postmortem morphometric study of schizophrenia and Huntington disease. Arch Gen Psychiatry 55:215-224

Rajkowska G, Miguel-Hidalgo JJ, Wei J, Dilley G, Pittman SD, Meltzer HY, et al. (1999): Morphometric evidence for neuronal and glial prefrontal cell pathology in major depression. Biol Psychiatry 45:1085-1098

Ravid R, Van Zweiten EJ, Swaab DF (1992): Brain banking and the human hypothalamus-factors to match for, pitfalls and potentials. Prog Brain Res 93:83-95

Sokolov BP, Tcherepanov AA, Haroutunian V, Davis KL (2000): Levels of mRNAs encoding synaptic vesicle and 
synaptic plasma membrane proteins in the temporal cortex of elderly schizophrenic patients. Biol Psychiatry 48:184-196

Stevens JR (1973): An anatomy of schizophrenia? Arch Gen Psychiatry 29:177-189

Vawter M, Crook JM, Hyde TM, Kleinman JE, Weinberger DR, Becker KG, Freed WJ (2001): Microarray analysis of gene expression in the prefrontal cortex in schizophrenia: A preliminary study. Schiz Research, in press

Volk DW, Austin MC, Pierri JN, Sampson AR, Lewis DA (2000): Decreased GAD67 mRNA expression in a subset of prefrontal cortical GABA neurons in subjects with schizophrenia. Arch Gen Psychiatry 57:237-245
West MJ (1999): Stereological methods for estimating total number of neurons and synapses: Issues of precision and bias. Trends Neurosci 22:51-61

Woo T-U, Whitehead RE, Melchitzky DS, Lewis DA (1998): A subclass of prefrontal gamma-aminobutyric acid axon terminals are selectively altered in schizophrenia. Proc Natl Acad Sci USA 95:5341-5346

Yates CM, Butterworth J, Tennant MC, Gordon A (1990): Enzyme activities in relation to $\mathrm{pH}$ and lactate in post mortem brain in Alzheimer type and other dementias. J Neurochem 55:1624-1630

Young KA, Manaye KF, Liang C-L, Hicks PB, German DC (2000): Reduced number of mediodorsal and anterior thalamic neurons in schizophrenia. Biol Psychiatry 47:944-953 